\title{
Effect of sacral slope on the biomechanical behavior of the low lumbar spine
}

\author{
YUGANG JIANG $^{1,2^{*}}$, XIAOJIANG SUN ${ }^{3 *}$, XIONGQI PENG ${ }^{1}$, JIE ZHAO $^{3}$ and KAI ZHANG ${ }^{3}$ \\ ${ }^{1}$ School of Materials Science and Engineering, Shanghai Jiaotong University, Shanghai 200030; \\ ${ }^{2}$ School of Construction Machinery, Shandong Jiaotong University, Jinan, Shandong 250000; ${ }^{3}$ Department of Orthopedics, \\ Ninth People's Hospital, School of Medicine, Shanghai Jiaotong University, Shanghai 200011, P.R. China
}

Received September 9, 2015; Accepted January 13, 2017

DOI: $10.3892 /$ etm.2017.4251

\begin{abstract}
The present study investigated the influence of sacral slope (SS) on the biomechanical responses of the lumbar spine under specific physiological conditions. Firstly, based on computed tomography scan images of a 30-year-old healthy male volunteer $\left(\mathrm{SS}, 55^{\circ}\right.$ ), a three-dimensional finite element (FE) model including the L4-S1 segment was established. Flexion, extension, lateral bending and torsion motions were simulated and compared with cadaveric test data in the literature to validate the lumbar spine FE model. The model was then modified with different SS values $\left(40\right.$ and $25^{\circ}$ ) for the same simulations to describe the process of structural compensation. Numerical results showed that with the reduction of SS, the range of motions (ROMs) reduced for flexion and lateral bending, but increased for extension and torsion. For displacement, the maximum magnitudes of L4/5 annulus fibrosus (AF) reduced by $10-25 \%$ in flexion, lateral bending and torsion, but less effect was observed for extension with only a $4 \%$ drop. Nearly the same displacement distribution appeared on the L5/S1 AF with small changes in the four motions. For the stress field of L4/5 AF, in contrast to flexion, the magnitudes for extension and lateral bending varied markedly, and under torsion the value increased by $\sim 10 \%$. For L5/S1 AF, the stresses changed little under flexion, extension and lateral bending, but strongly declined for torsion by $\sim 71.8 \%$. In conclusion, the present study indicates that the change in SS due to structural compensation affects the biomechanical behavior of the spine structure, and attention should be paid to SS when conducting surgical procedures or selecting intervertebral fusion implants.
\end{abstract}

Correspondence to: Professor Xiongqi Peng, School of Materials Science and Engineering, Shanghai Jiaotong University, 1954 Huashan Road, Xuhui, Shanghai 200030, P.R. China

E-mail: xqpeng@sjtu.edu.cn

${ }^{*}$ Contributed equally

Key words: biomechanics, finite element method, lumbar spine, sacral slope

\section{Introduction}

The human low lumbar spine and pelvis compromise a complex system that is required for the maintenance of balance. For sagittal balance, several parameters, including pelvic incidence (PI), pelvic tilt (PT) and sacral slope (SS) (1-3) have been defined to evaluate the alignment of the spino-pelvic complex. These parameters cooperate to regulate sagittal balance between the spine and pelvis in order to keep an individual standing in an erect position (4). PI is the angle created by the intersection of a line drawn from the center of the femoral heads to the middle of the sacral plate and a line running perpendicular to the middle of the sacral plate, and PT is the angle between the vertical and a line drawn from the center of the femoral heads to the center of the upper sacral endplate. PI is a morphological constant for an individual in adulthood (4). However, the SS, which is defined as the angle between the superior plate of S1 and a horizontal line as shown in Fig. 1, is a parameter changing with age (4). The geometric relation between PI, PT and SS is as follows: $\mathrm{PI}=\mathrm{PT}+\mathrm{SS}$. When the lumbar spine endures intervertebral disc degeneration or certain other spinal diseases, it may lose lordosis; however, the spine-pelvis complex has the capability to compensate for sagittal imbalance of the spine through pelvic retroversion with changing SS (4). The change of SS secondary to reduction of lumbar lordosis affects the biomechanics of the lumbar spine and results in a deterioration of sagittal balance.

The biomechanical response of the human lumbar spine has been investigated via experimental or computational approaches for decades. However, SS may be an ignored or rejected factor in these two approaches for various reasons. Above all, it is difficult to determine the SS parameter from experimentation using cadaver samples. Therefore, the data obtained from in vitro experiments is not able to clearly indicate the variation in biomechanical behavior that results from a changing SS (5-10). In computational analysis using a finite element method (FEM), magnitudes and distributions of displacements, stresses and range of motion (ROM) under different daily physical motions can be provided. However, few studies (11-15) have considered the effect of a changing SS caused by structural compensation. Furthermore, if the influence of SS on ROMs, stress and strain distribution of the spine is known, this may be beneficial in the preparation of 
personalized fusion implant designs and therapeutic schedules. Hence, for focusing on investigating the biomechanical behaviors of the low lumbar spine segment with a changing SS caused by structural compensation, a computational model with the capability for parametric study is required so that complex in vivo and in vitro experiments are not necessary. In the present study, a three-dimensional finite element (FE) model including the L4-S1 segment was developed, then modified with different SS. Following model validation, four daily physical motions including flexion, extension, lateral bending and torsion were simulated to predict the alteration of biomechanical responses under a changing SS.

\section{Materials and methods}

Lumbar finite element model (L4-S1). In an FEM, a geometrical complex spine segment can be divided into different regions according to its anatomical structure, and then meshed with various types of elements. Each region can be assigned an appropriate material model to reflect its biomechanical characteristics. In the present study, a surface model (Fig. 2A) including the L4-S1 segment was first constructed based on computed tomography (CT) scan images of a 30 -year-old healthy male volunteer with an SS of $55^{\circ}$. A corresponding solid model was then constructed in HyperMesh (Altair Engineering, Inc., Troy, MI, USA). Meshing was also implemented in HyperMesh (Fig. 2B). Finally, biomechanical finite element analysis of the L4-S1 segment was carried out in ABAQUS/Standard (Dassault Systèmes, Vélizy-Villacoublay, France). The model included the vertebrae, intervertebral discs, endplates and ligaments.

As shown in Fig. 3, the vertebrae were divided into cortical and cancellous bones represented by an outer layer of hexahedral solid elements and enclosed tetrahedral solid elements, respectively. The thickness of the cortical bones was assumed to be $1 \mathrm{~mm}$ (16). Notably, the nodes were shared at the interface between cortical and cancellous bones to avoid complex interaction problems. Bones usually exhibit a highly non-linear biomechanical response under high loading conditions such as impact and bone fracture (17). In current study, as the focus was on the biomechanical behavior of the lumbar spine under daily physiological motions, the cortical and cancellous bones were assumed to be homogeneous and isotropic materials with different elastic constants, as listed in Table I (18). Contact surfaces with a distance of $0.5 \mathrm{~mm}$ were defined to simulate the facet joints (19).

The intervertebral discs were divided into superior and inferior endplates, annulus fibrosus and nucleus pulposus. The endplates had a thickness of $0.5 \mathrm{~mm}(20)$ and were connected with their adjacent vertebrae by sharing common nodes on the interfaces. They were meshed by 3D solid elements and assigned a linear isotropic elastic model with material parameters listed in Table I. For the disc, 30-50\% of the cross-section area was defined as nucleus, and the rest was processed as the annulus fibrosus (21). The nucleus was assumed to be a nearly incompressible material by assignment of a Poisson's ratio of 0.499 and a low Young's modulus of $1 \mathrm{MPa}$. The ROM of the human spine is mainly affected by intervertebral discs. The constitutive model for large deformation segments such as the annulus fibrosus is considered to provide accurate results.

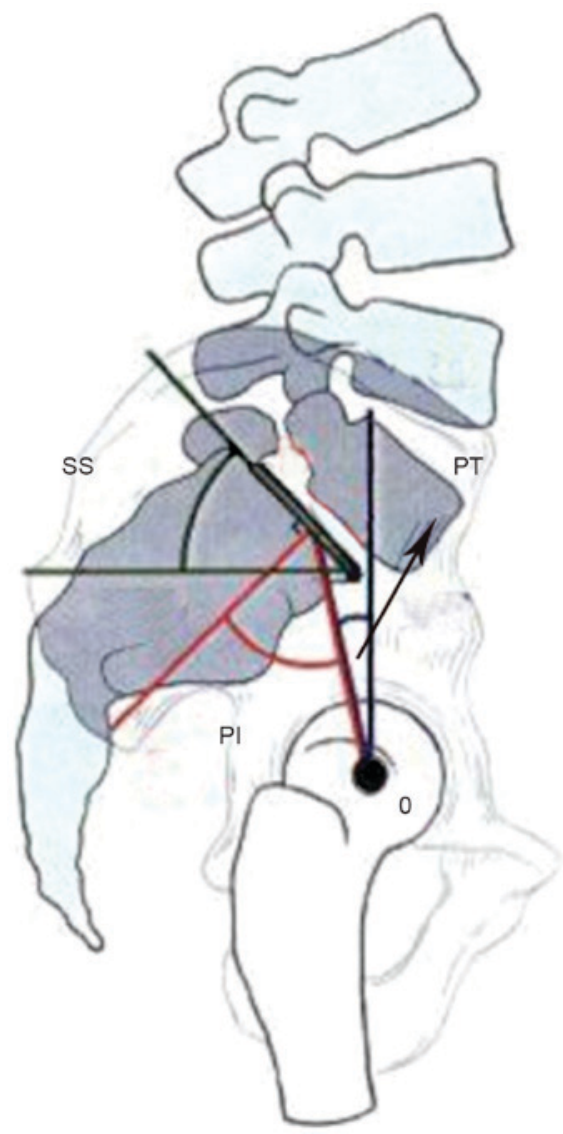

Figure 1. Illustration of SS, PI and PT. SS, sacral slope; PI, pelvic incidence; PT, pelvic tilt.

The annulus fibrosus is often characterized as fiber-reinforced materials in which several matrix layers are embedded with rebar elements representing collagen fibers $(11,12,14,15,22)$. The effect of interaction between fibers and matrix is ignored when using the one-dimensional rebar elements method. Also, this method increases meshing difficulty. To overcome these shortcomings, Peng et al (23) developed a continuum mechanics-based fiber reinforced hyperelastic model to characterize the anisotropic nonlinear biomechanical behavior of annulus fibrosus. The strain energy function to determine the constitutive relationship is given as follows:

$$
W=W^{M}+W^{F}+W^{F M}
$$

where $W^{\mathrm{M}}, W^{\mathrm{F}}$ and $W^{\mathrm{FM}}$ are the energy contribution from ground matrix, fiber elongation and the interaction between matrix and fibers, respectively. The specific forms of strain energy functions for the annulus fibrosis are given as follows:

$$
\begin{aligned}
& W^{M}=C_{10}\left(\bar{I}_{1}-3\right)+\frac{1}{D_{1}}(J-1)^{2} \\
& W^{F}=\left\{\begin{array}{cc}
0 & I_{4} \leq 1 \\
C_{2}\left(I_{4}-1\right)^{2}+C_{3}\left(I_{4}-1\right)^{4} I_{4}>1
\end{array}\right. \\
& W^{F M}=W^{F M}\left(I_{4}, \phi\right)=f\left(I_{4}\right) \chi^{2}=f\left(I_{4}\right)\left[\frac{I_{4}}{I_{3}}\left(I_{5}-I_{1} I_{4}+I_{2}\right)-1\right]^{2}
\end{aligned}
$$

where $\left(I_{4}\right)=\frac{\gamma}{1+\exp \left[-\beta\left(\lambda_{F}-\lambda_{F}^{*}\right)\right]}$ and $I_{i}(i=1, \ldots, 5)$ are principal invariants, and $C_{10}=0.034 \mathrm{MPa}, D_{1}=0.197 \mathrm{MPa}^{-1}$, 
Table I. Properties of materials.

\begin{tabular}{lcccc}
\hline Material & Young's modulus E $(\mathrm{MPa})$ & Poisson's ratio $v$ & Element type & (Refs.) \\
\hline Vertebrae & & & & \\
Cortical bone & 12,000 & 0.3 & C3D8 & $(18,25-30)$ \\
Cancellous bone & 100 & 0.2 & C3D 4 & $(18,27-30)$ \\
Endplate & 12,000 & 0.3 & C3D8 & $(26,28,31)$ \\
Disc & & & & \\
Nucleus & 1 & 0.499 & C3D & $(29,32-34)$ \\
Annulus fibrosus & User defined material & User defined material & C3D 8 & $(23)$ \\
Ligaments & & & & $(35,36)$ \\
ALL & 7.8 & 0.3 & S4 & $(35,36)$ \\
PLL & 10 & 0.3 & S4 & $(35,36)$ \\
LF & 15 & 0.3 & S4 & $(35,36)$ \\
CL & 7.5 & 0.3 & S4 & $(35,36)$ \\
ISL & 8 & 0.3 & S4 & $(35,36)$ \\
SSL & 8 & 0.3 & & \\
\hline
\end{tabular}

ALL, anterior longitudinal ligament; PLL, posterior longitudinal ligament; LF, ligamentum flavum; CL, capsular ligament; ISL, interspinous ligament; SSL, supraspinal ligament.
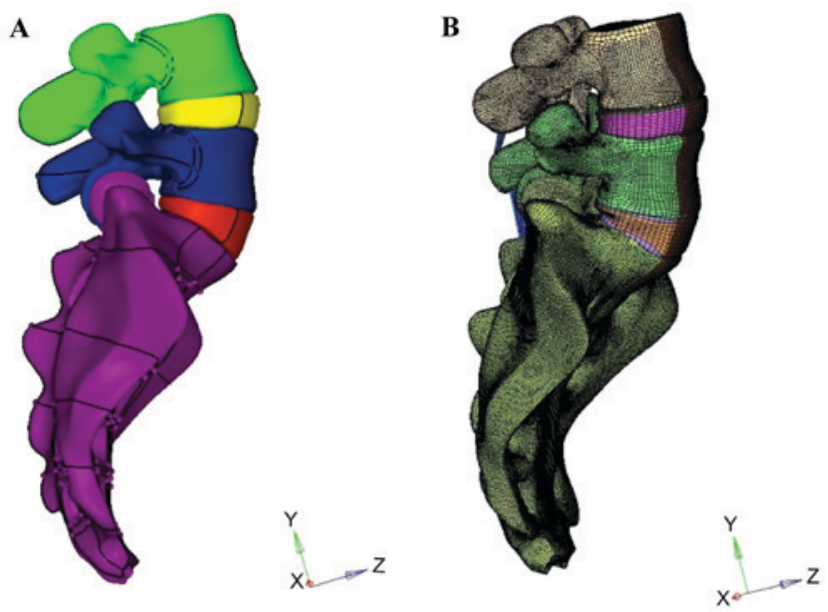

Figure 2. (A) Surface and (B) finite element models for the L4-S1 lumbar spine segment.

$C_{2}=0.45 \mathrm{MPa}, C_{3}=82.6 \mathrm{MPa}, \gamma=12.0 \mathrm{MPa} \beta=125$ and $\lambda_{\mathrm{F}}^{*}=1.02$ are material parameters. More detail has been provided in a previous study (23). This constitutive model was implemented by designing a user defined material subroutine (UANISOHYPER) in ABAQUS/Standard (Dassault Systèmes). The orientation of fibers was defined as $\pm 30^{\circ}$ to the horizontal plane $(13,23)$.

Ligaments play a major role in spinal stability and function (24). A total of 6 ligaments including the anterior longitudinal ligament (ALL), posterior longitudinal ligament (PLL), ligamentum flavum (LF), capsular ligament (CL), interspinous ligament (ISL) and supraspinal ligament (SSL) were modeled as isotropic linear elastic membranes that are able to bear tensile loads only.

Material parameters for different parts of the model are summarized in Table I.

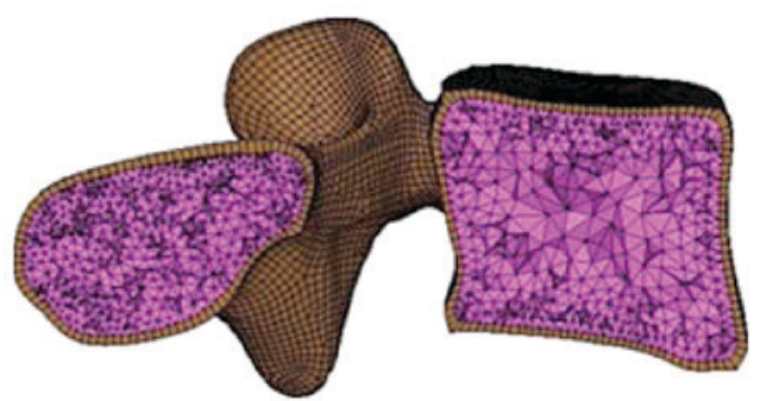

Figure 3. Sketch map of cortical and cancellous bones (L4). The cortical and cancellous bones are represented by an outer layer of hexahedral solid elements and enclosed tetrahedral solid elements, respectively.

L4-S1 models with different SS. In order to investigate the influence of SS on the biomechanical behavior of the lumbar spine, the L4-S1 spine model was modified with different SS. In accordance with a previous study (4), the SS angles were divided into three groups: $\mathrm{SS}>45^{\circ}, 35^{\circ}<\mathrm{SS} \leq 45^{\circ}$ and $\mathrm{SS} \leq 35^{\circ}$. As shown in Fig. 4, three SS angles of 55, 40 and $25^{\circ}$ were chosen as the typical angle for each group. Noting that the change of lumbar lordosis causes pelvis retroversion, then influences the SS angle, the shape of the intervertebral discs was slightly changed as shown in Fig. 4. For convenience, models I, II and III are used for distinction. Convergence testing was performed to filter the influence of mesh size. The model had 260,729 nodes and 854,845 elements with an average element size of $\sim 0.75 \mathrm{~mm}$.

Boundary and loading conditions. Considering the daily physiological actions of the human spine, four basic motions including flexion, extension, lateral bending and torsion were selected in numerical simulations. S5 were completely constrained. According to the study by Yamamoto et al (9), 

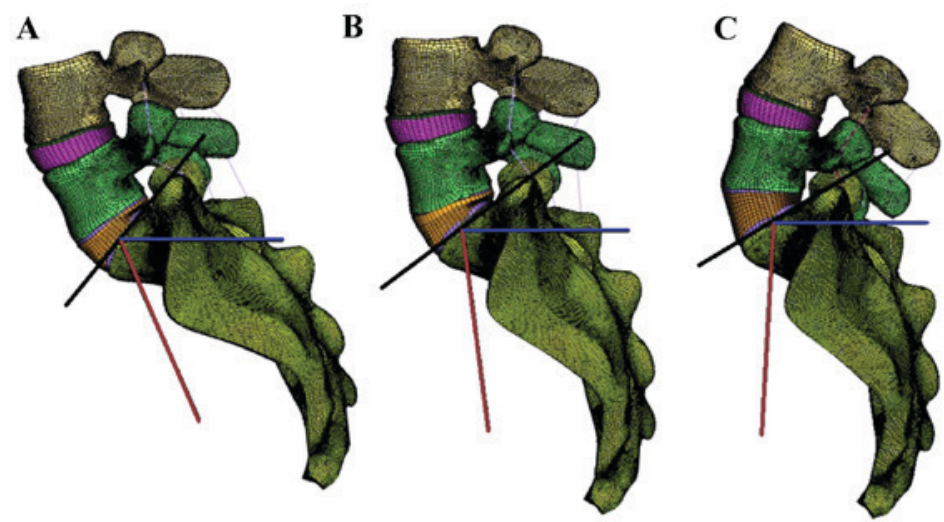

Figure 4. L4-S1 models with different SS angles. The models have SS values of (A) $55^{\circ}$, (B) $40^{\circ}$ and (C) $25^{\circ}$. The blue line is a reference line across the center of the upper sacral endplate. The red line links the center of the femoral heads and the center of the upper sacral endplate. The black line is a tangent line across the center of the upper sacral endplate. SS, sacral slope.

A
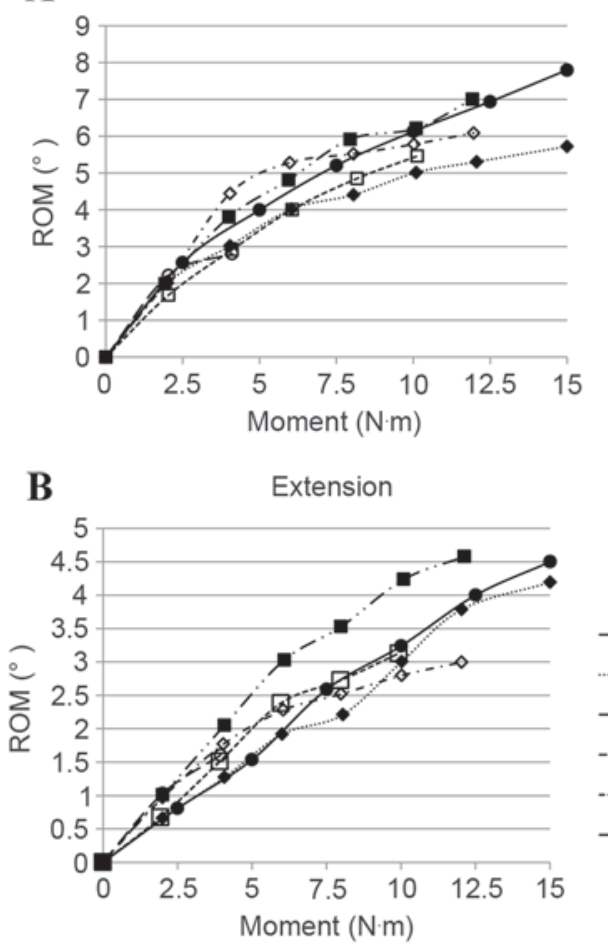

$-\Theta-$ Markolf (5)

$\bullet . . .$. Shirazi-Adl (6)

- - - Tencer (7)

- • - Schultz (8)

-- --- Chen (36)

$\longrightarrow$ Present study

Figure 5. ROMs of flexion and extension with different loading magnitudes. (A) Flexion and (B) extension. ROM, range of motion.

a $150-\mathrm{N}$ vertical axial pre-load was imposed on the superior surface of L4; at the same time, a 10-N·m moment was applied on the L4 superior surface along the radial direction to simulate the four basic physiological motions.

Simulation results of the L4-S1 model with an SS of $55^{\circ}$ under various pathological loading conditions were compared with cadaveric test data in the literature for model validation. Then, numerical simulations were extended to other L4-S1 models with different SS values to investigate the effect on the biomechanical responses of the lumbar spine.

Model validation. For model validation, ROMs under different human physiological motions were employed as a verification criterion. Table II lists the predicted ROMs of the L4-S1 FE model under flexion, extension, lateral bending and torsion, respectively with a $150-\mathrm{N}$ pre-load and a $10-\mathrm{N} \cdot \mathrm{m}$ moment. For comparison, in vitro ROMs reported by different research groups $(6-9,36)$ are also presented in Table II.

As shown in Table II, the in vitro ROMs reported by different groups vary in a range of $4.9-6.2^{\circ}$ for flexion, and $2.8-4.2^{\circ}$ for extension. In the present study, the ROM was 6.13 and $3.24^{\circ}$ for flexion and extension, respectively. The simulation results fall into the in vitro experimental ranges of ROMs for the two motions, respectively. For lateral bending and torsion, the predicted ROMs were 3.47 and $1.79^{\circ}$, respectively, a little bit below the in vitro experimental ranges of ROMs, which are $3.5-4.2^{\circ}$ for lateral bending and $2-2.8^{\circ}$ for torsion (9).

Fig. 5 shows the in vitro experimental ROMs of the L4-S1 lumbar spine segment under flexion and extension with different loading magnitudes from 0 to $15 \mathrm{~N} \cdot \mathrm{m}$, respectively $(5-8,36)$. Numerical results of the present model are also shown in Fig. 5A for flexion and Fig. 5B for extension by solid lines with filled circles. As presented in Fig. 5, the simulation results are in concordance with the experimental data, bearing in mind the deviations of in vitro testing data from different studies. The ROMs are nonlinear to the applied moment and render a decreasing tendency with the increase of applied moment for both motions.

\section{Results and Discussion}

With the lumbar spine FE model being validated, FE analyses on L4-S1 models with an SS of $40^{\circ}$ (model II) and $25^{\circ}$ (model III) were carried out for the four basic physiological motions to investigate the effects of SS.

As shown in Table II, under flexion with a $10-\mathrm{N} \cdot \mathrm{m}$ moment, the predicted ROMs for the two models were 5.70 and $5.34^{\circ}$, respectively. Compared with model I which had an SS angle of $55^{\circ}$, the ROMs of model II and III were decreased by 7.01 and $12.8 \%$, respectively. By contrast, the predicted ROMs increased with the reduction of SS angle for extension, lateral bending and torsion, as indicated in Table II. However, almost all numerical ROMs for the four motions from the three models fell into the in vitro experimental ranges of ROMs. 
Table II. Range of motion $\left({ }^{\circ}\right)$ for different motions.

\begin{tabular}{|c|c|c|c|c|c|}
\hline Study & Flexion & Extension & Lateral bending & Torsion & (Ref.) \\
\hline Shirazi-Adl et al & 4.96 & 2.94 & - & - & $(6)$ \\
\hline Tencer et al & 6.23 & 4.19 & - & - & $(7)$ \\
\hline Schultz et al & 5.79 & 2.79 & - & - & (8) \\
\hline Chen et al & 5.41 & 3.13 & - & - & (36) \\
\hline Yamamoto et al & - & - & $3.5-4.2$ & $2-2.8$ & (9) \\
\hline \multicolumn{6}{|l|}{ Present study } \\
\hline $\mathrm{SS}=55^{\circ}$ & 6.13 & 3.24 & 4.07 & 1.79 & \\
\hline $\mathrm{SS}=40^{\circ}$ & 5.70 & 3.50 & 3.72 & 2.09 & \\
\hline $\mathrm{SS}=25^{\circ}$ & 5.34 & 3.89 & 3.79 & 2.20 & \\
\hline
\end{tabular}

SS, sacral slope.
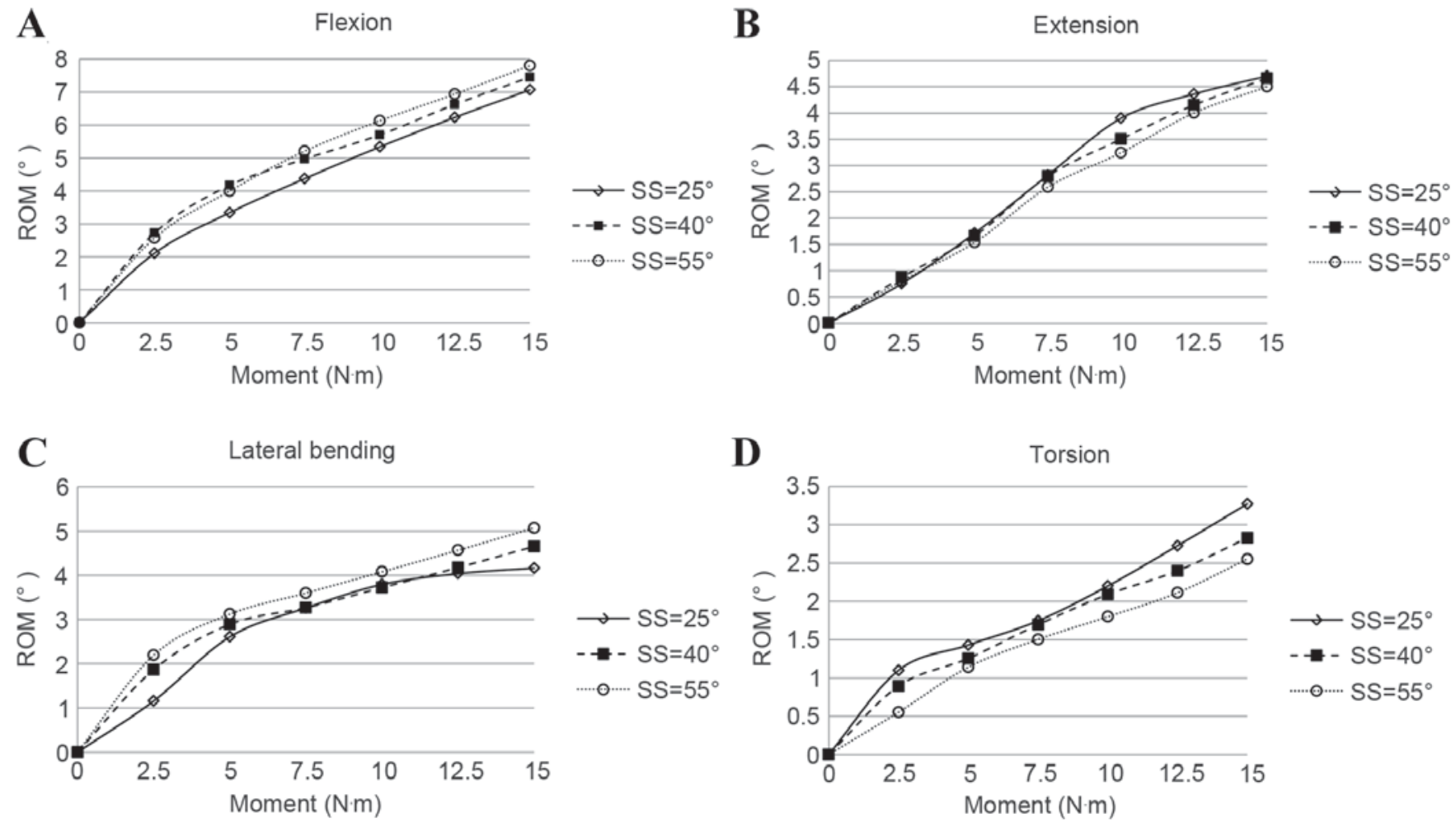

Figure 6. ROMs of the four motions with different loading moments. (A) Flexion, (B) extension, (C) lateral bending and (D) torsion. ROM, range of motion; SS, sacral slope.

The ROMs of the three models under the four motions with different loading moment magnitudes are presented in Fig. 6. Generally, the spine segment showed an increased stiffness with respect to ROM with the increase of loading moment for all four motions. Under the same moment, the ROM increased with the increase of SS for flexion and lateral bending; under extension and torsion, the opposite trend occurred.

As shown in Table II and Fig. 6, the variation of ROMs under the same loading condition with different SS was distinct. The maximum difference of ROM was $>10 \%$ for the four motions. Therefore, the SS can influence the biomechanical behavior of the spine markedly.
The SS affected not only the ROMs, but also the displacement distribution of annulus fibrosus in the spine segment. Fig. 7 shows the displacement distribution in the L4/5-disc annulus under a $150-\mathrm{N}$ pre-load and $10-\mathrm{N} \cdot \mathrm{m}$ moment for the four motions with an SS of $55^{\circ}$. For flexion, the maximum displacement was found at the border close to the upper vertebrae segment with a magnitude of $\sim 2.0 \mathrm{~mm}$ as shown in Fig. 7A. At nearly the same position, the displacement increased to $2.4 \mathrm{~mm}$ for extension (Fig. 7B). The value gradually increased and reached $3.4 \mathrm{~mm}$ at the compression region under lateral bending detailed in Fig. 7C, while the peak magnitude in all four motions occurred in torsion $\sim 5.0 \mathrm{~mm}$ as presented in Fig. 7D. 


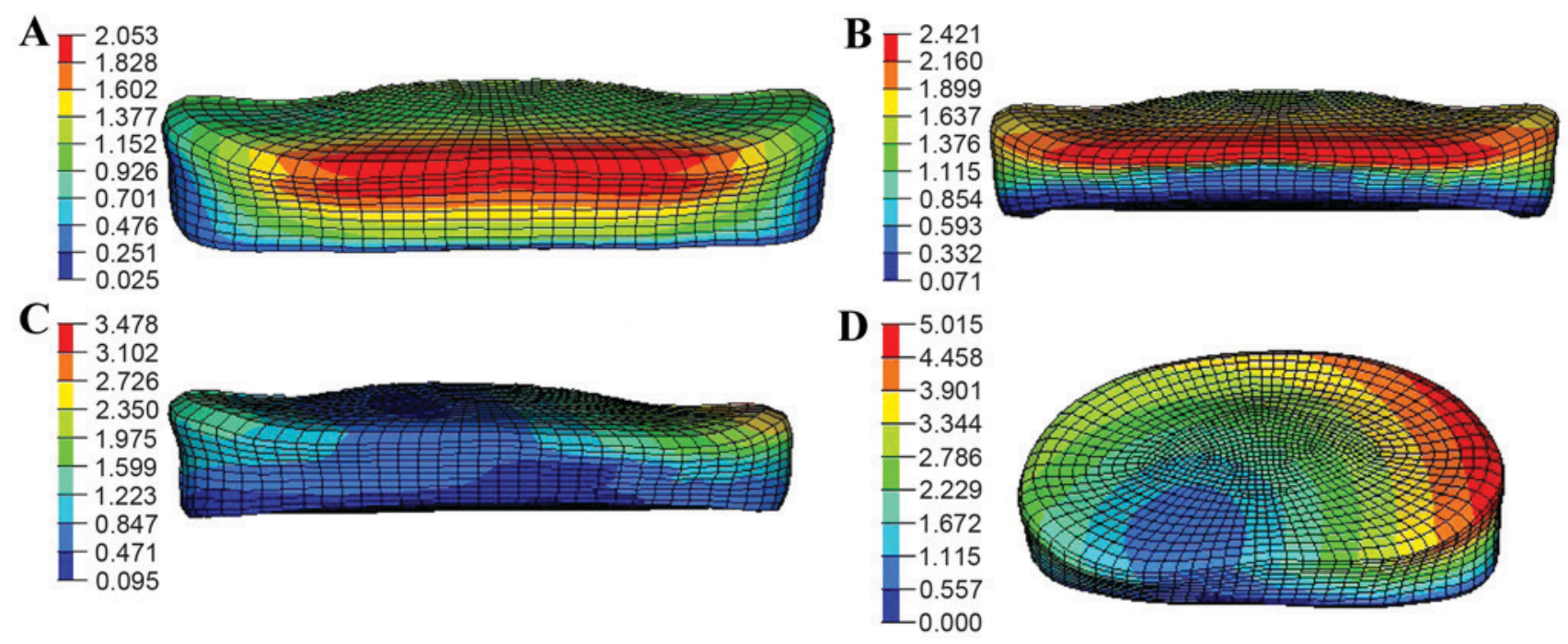

Figure 7. Displacement field distribution of four motions (L4/5 disc with a sacral slope of $55^{\circ}$ ). (A) Flexion, (B) extension, (C) lateral bending and (D) torsion.

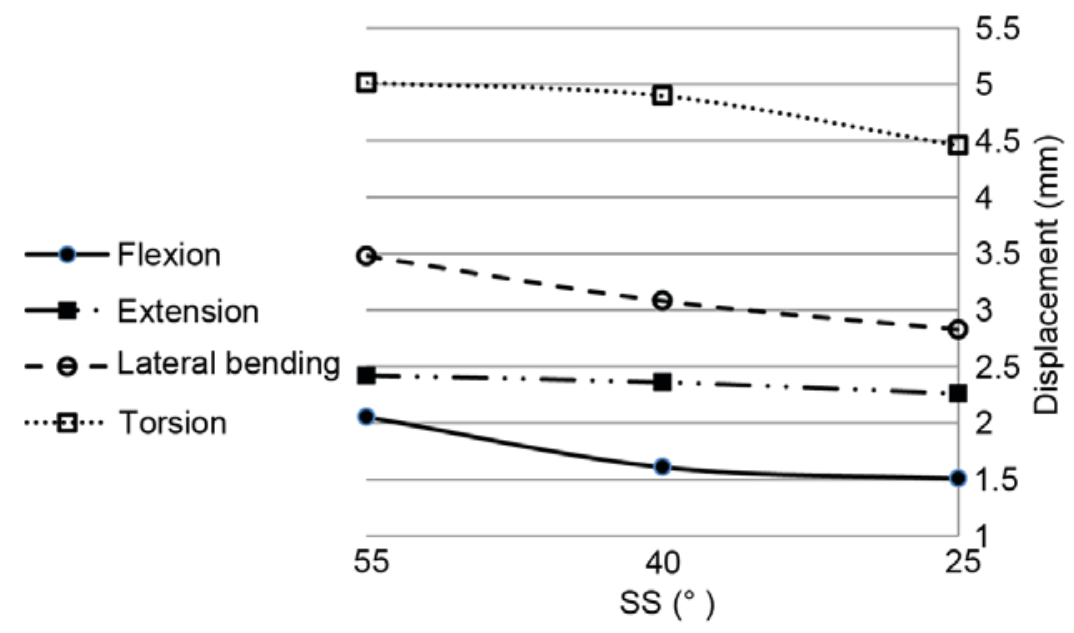

Figure 8. Predicted maximum displacement in different SS models (L4/5 annulus fibrosus). SS, sacral slope.

The changes of the maximum displacements in the L4/5 annulus fibrosus for the four motions with the change of SS are shown in Fig. 8. When SS decreased, the maximum displacement decreased for all four motions. The maximum reduction rate was $\sim 10,14.3$ and $25 \%$ for flexion, lateral bending and torsion, respectively, but only $4 \%$ for extension. When intervertebral discs degenerate with loss of lumbar lordosis, adjacent lumbar segments are likely to compensate with the increase of lordosis, followed by retroversion of the pelvis with the reduction of SS, with reduction of the displacement of the annulus fibrosus. Additionally, although it was not shown, nearly the same displacement distribution appeared for the L5/S1 disc with maximum values of only $\sim 0.8,0.5,1.3$ and $2.2 \mathrm{~mm}$ for flexion, extension, lateral bending and torsion, respectively.

The von Mises stress distribution on the L4/5 annulus fibrosus under a $150-\mathrm{N}$ pre-load and $10-\mathrm{N} \cdot \mathrm{m}$ moment is shown in Fig. 9. The largest stress region appeared in the posterior side of the annulus fibrosus, particularly in flexion, extension and lateral bending (Fig. 9A-C). For torsion, the annulus fibrosus was twisted, so the stress mainly distributed along the orientation of fibers with a maximum magnitude of $\sim 4.9 \mathrm{MPa}$ as detailed in Fig. 9D.

The changes of the maximum von Mises stress in the L4/5 annulus fibrosus for the four motions with the change of SS are shown in Fig. 10. With the reduction of SS from 55 to $25^{\circ}$, the maximum von Mises stresses for extension markedly increased from 1.3 to $2.4 \mathrm{MPa}$, an increase of $\sim 84 \%$. For lateral bending, the maximum von Mises stress decreased from 3.2 to $2.2 \mathrm{MPa}$, a reduction of $\sim 30 \%$. For flexion, the effect of SS was insignificant. The maximum von Mises stress fluctuated by $\sim 3.0 \mathrm{MPa}$. For torsion, the maximum stress initially increased then decreased with the reduction of SS 5.3 MPa. For the L5/S1 disc, the stresses had little change under flexion, extension and lateral bending with magnitudes of $\sim 1.3,0.1$ and $1.6 \mathrm{MPa}$, respectively. However, for torsion, the stresses declined notably, and were 3.2, 1.7 and $0.9 \mathrm{MPa}$ in the three models.

Briefly, when an intervertebral disc degenerates, the spine structure compensates to keep sagittal balance by retroversion of the pelvis with reduction of the SS. The predictions based on FEM show that as SS decreased, the ROMs of flexion and lateral bending reduced, and the ROMs of extension 
A

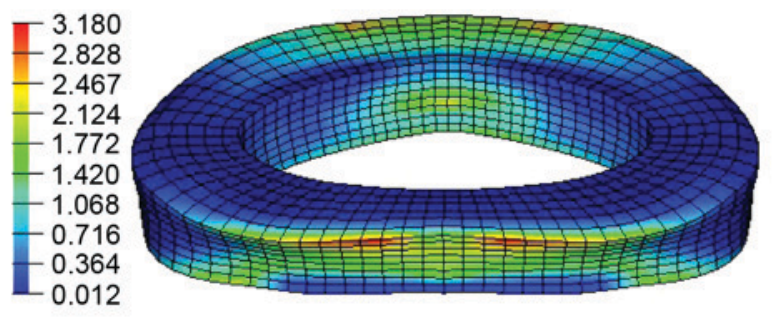

B

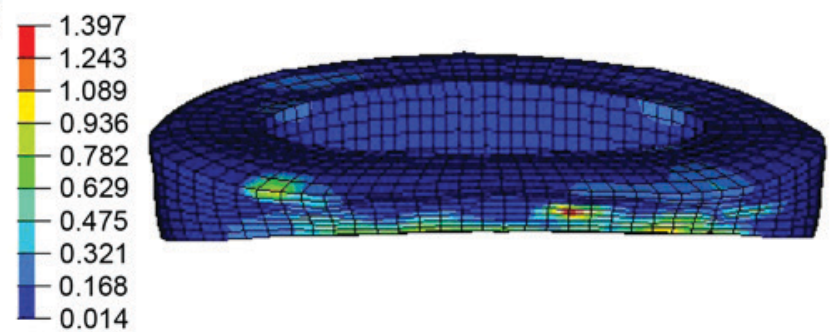

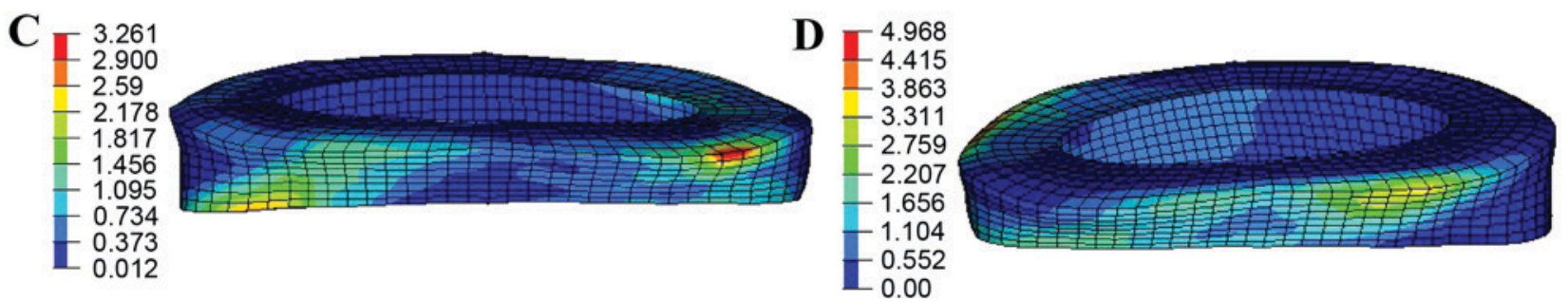

Figure 9. Von Mises stress distribution at L4/5 annulus fibrosus. (A) Flexion, (B) extension, (C) lateral bending and (D) torsion.

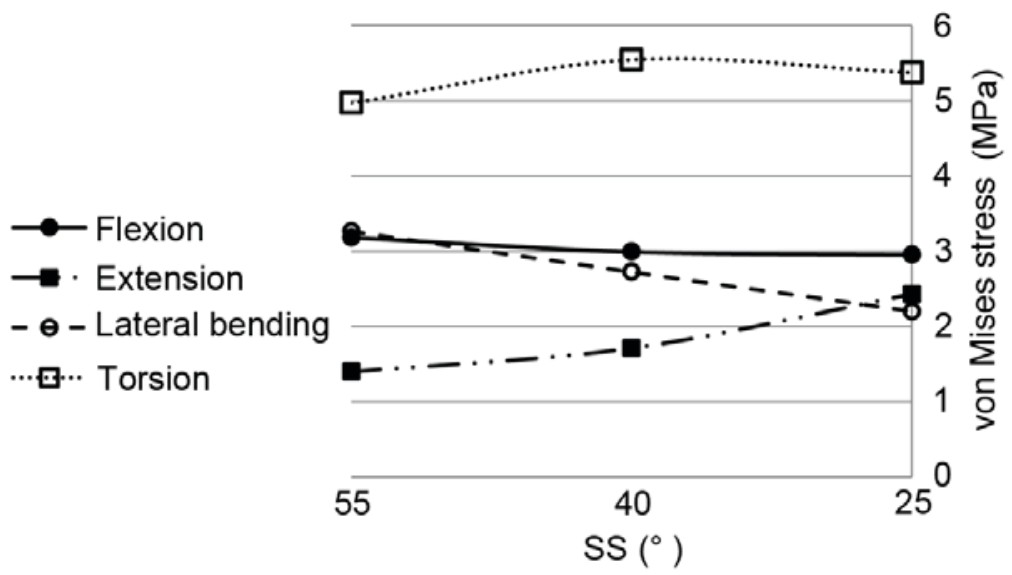

Figure 10. Predicted maximum von Mises stress in different SS models (L4/5 annulus fibrosus). SS, sacral slope.

and torsion increased. The maximum displacements reduced $10-25 \%$ in flexion, lateral bending and torsion, but in extension the maximum displacement only dropped $4 \%$. Considering stress, in contrast to flexion, the magnitudes for extension and lateral bending varied markedly, and for torsion the value increased by $\sim 10 \%$ initially, then decreased with the reduction of SS. Although only the L4 to S1 segment was considered in the present model, the mechanical responses including ROMs, displacement and stress had different degrees of variation, according to this study, when compensation occurred; thus, the SS did have a great influence on the biomechanical behavior of the spine structure.

The present study has several limitations. Firstly, the material models for ligaments and vertebrae were simplified and idealized. Secondly, spine structural compensation often occurs following disc degeneration (4). However, the present study ignored the effect of disc degeneration and only focused on the influence of SS. Thirdly, structural compensation also influences regions other than the L4-S segment, particularly for intervertebral discs (4). Therefore, the current spinal model should be extended by including more spinal segments. Lastly, in the human body, vertebrae are connected by not only ligaments, but also by muscles, which was ignored in the current model. Our future studies will concentrate on developing a more accurate model to comprehend the biomechanical essentials of human spine segment.

In conclusion, in the present study a three-dimensional finite element model of L4-S spine was established to investigate the effect of SS on biomechanical behavior under various daily actions, including flexion, extension, lateral bending and torsion motions. The model was validated by comparing simulation results with in vitro cadaveric test data. The numerical predictions indicated that as SS decreased, the ROMs of flexion and lateral bending reduced, and the ROMs of extension and torsion increased. The maximum displacements of L4/5 AF reduced 10-25\% in flexion, lateral bending and torsion, whereas the displacement of extension dropped only 4\%. The effect of SS on von Mises stress of L4/5 AF was minimal in flexion, but marked in lateral bending with a $31 \%$ drop. By contrast, the stress increased $\sim 84 \%$ in extension. In torsion, the maximum stress initially increased then decreased with the reduction of SS. The present study indicated that the change of SS caused by structural compensation affected the biomechanical behavior of the spine structure, and attention 
should be paid to SS when conducting surgical procedures or selecting intervertebral fusion implants.

\section{Acknowledgements}

Support from the National Natural Science Foundation of China (grant no. 11172171) and the Science and Technology Commission of Shanghai Municipality (grant nos. 13DZ1940504 and 13DZ1940505) are gratefully acknowledged.

\section{References}

1. Duval-Beaupère G, Schmidt C and Cosson P: A barycentremetric study of the sagittal shape of spine and pelvis: The conditions required for an economic standing position. Ann Biomed Eng 20: 451-462, 1992

2. Duval-Beaupère $\mathrm{G}$ and Legaye $\mathrm{J}$ : Composante sagittale de la statique rachidienne. Revue Du Rhumatisme 71: 105-119, 2004.

3. Boulay C, Tardieu C, Hecquet J, Benaim C, Mouilleseaux B, Marty C, Prat-Pradal D, Legaye J, Duval-Beaupère G and Pélissier J: Sagittal alignment of spine and pelvis regulated by pelvic incidence: Standard values and prediction of lordosis. Eur Spine J 15: 415-422, 2006.

4. Roussouly P and Pinheiro-Franco JL: Biomechanical analysis of the spino-pelvic organization and adaptation in pathology. Eur Spine J 20 (Suppl 5): S609-S618, 2011.

5. Markolf KL: Deformation of the thoracolumbar intervertebral joints in response to external loads: A bomechanical study using autopsy material. J Bone Joint Surg Am 54: 511-533, 1972 .

6. Shirazi-Adl SA, Shrivastava SC and Ahmed AM: Stress analysis of the lumbar disc-body unit in compression. A three-dimensional nonlinear finite element study. Spine (Phila Pa 1976) 9: 120-134, 1984

7. Tencer AF, Ahmed AM and Burke DL: Some static mechanical properties of the lumbar intervertebral joint, intact and injured. J Biomech Eng 104: 193-201, 1982.

8. Schultz A, Andersson G, Ortengren R, Haderspeck K and Nachemson A: Loads on the lumbar spine. Validation of a biomechanical analysis by measurements of intradiscal pressures and myoelectric signals. J Bone Joint Surg Am 64: 713-720, 1982

9. Yamamoto I, Panjabi MM, Crisco $\mathrm{T}$ and Oxland $\mathrm{T}$ : Three-dimensional movements of the whole lumbar spine and lumbosacral joint. Spine (Phila Pa 1976) 14: 1256-1260, 1989.

10. Shirazi-Adl A and Pamianpour M: Nonlinear response analysis of the human ligamentous lumbar spine in compression. On mechanisms affecting the postural stability. Spine (Phila Pa 1976) 18: $147-158,1993$

11. Kuo CS, Hu HT, Lin RM, Huang KY, Lin PC, Zhong ZC and Hseih ML: Biomechanical analysis of the lumbar spine on face joint force and intradiscal pressure-a finite element study. BMC Musculoskelet Disord 11: 151, 2010.

12. Kim KT, Lee SH, Suk KS, Lee JH and Jeong BO: Biomechanical changes of the lumbar segment after total disc replacement: Charite(r), prodisc(r) and maverick(r) using finite element model study. J Korean Neurosurg Soc 47: 446-453, 2010.

13. Ruberté LM, Natarajan RN and Andersson GB: Influence of single-level lumbar degenerative disc disease on the behavior of the adjacent segments-a finite element model study. J Biomech 42: 341-348. 2009.

14. Chen SH, Zhong ZC, Chen CS, Chen WJ and Hung C: Biomechanical comparison between lumbar disc arthroplasty and fusion. Med Eng Phys 31: 244-253, 2009.

15. Chen SH, Tai CL, Lin CY, Hsieh PH and Chen WP: Biomechanical comparison of a new stand-alone anterior lumbar interbody fusion cage with established fixation techniques-a three-dimensional finite element analysis. BMC Musculoskelet Disord 9: 88, 2008.
16. Ritzel H, Amling M, Pösl M, Hahn M and Delling G: The thickness of human vertebral cortical bone and its changes in aging and osteoporosis: A histomorphometric analysis of the complete spinal column from thirty-seven autopsy specimens. J Bone Miner Res 12: 89-95, 1997.

17. Morgan EF, Yeh OC, Chang WC and Keaveny TM: Nonlinear behavior of trabecular bone at small strains. J Biomech Eng 123: $1-9,2001$.

18. Goel VK, Ramirez SA, Kong W and Gilbertson LG: Cancellous bone Young's modulus variation within the vertebral body of a ligamentous lumbar spine-application of bone adaptive remodeling concepts. J Biomech Eng 117: 266-271, 1995.

19. Zander T, Rohlmann A, Calisse J and Bergmann G: Estimation of muscle forces in the lumbar spine during upper-body inclination. Clin Biomech (Bristol, Avon) 16 (16 Suppl 1): S73-S80, 2001.

20. Silva MJ, Wang C, Keaveny TM and Hayes WC: Direct and computed tomography thickness measurements of the human, lumbar vertebral shell and endplate. Bone 15: 409-414, 1994.

21. Panagiotacopulos ND, Pope MH, Krag MH and Block R: Water content in human intervertebral discs. Part I. Measurement by magnetic resonance imaging. Spine (Phila Pa 1976) 12: 912-917, 1987.

22. Sairyo K, Goel VK, Vadapalli S, Vishnubhotla SL, Biyani A, Ebraheim N, Terai T and Sakai T: Biomechanical comparison of lumbar spine with or without spina bifida occulta. A finite element analysis. Spinal Cord 44: 440-444, 2006.

23. Peng XQ, Guo ZY and Moran B: An anisotropic hyperelastic constitutive model with fiber-matrix shear interaction for the human annulus fibrosus. J Appl Mech 73: 815, 2006.

24. Agur AMR and Lee MJ (eds): Grant's atlas of anatomy. 10th edition. Lippincott Williams \& Wilkins, Philadelphia, PA, 1999.

25. Polikeit A, Ferguson SJ, Nolte LP and Orr TE: Factors influencing stresses in the lumbar spine after the insertion of intervertebral cages: Finite element analysis. Eur Spine J 12: 413-420, 2003.

26. Pitzen T, Geisler FH, Matthis D, Müller-Storz H, Pedersen K and Steudel WI: The influence of cancellous bone density on load sharing in human lumbar spine: A comparison between an intact and a surgically altered motion segment. Eur Spine J 10: 23-29, 2001.

27. Natarajan R and Andersson G: Modeling the annular incision in a herniated lumbar intervertebral disk to study its effect on disk stability. Computers Structures 64: 1291-1297, 1997.

28. Denoziere G: Numerical modeling of a ligamentous lumbar motion segment. MSc Dissertation, Georgia Institute of Technology, 2004.

29. Sairyo K, Goel VK, Masuda A, Vishnubhotla S, Faizan A, Biyani A, Ebraheim N, Yonekura D, Murakami R and Terai T: Three-dimensional finite element analysis of the pediatric lumbar spine. Part I: Pathomechanism of apophyseal bony ring fracture. Eur Spine J 15: 923-929, 2006.

30. Panjabi MM, Oxland TR, Yamamoto I and Crisco JJ: Mechanical behavior of the human lumbar and lumbosacral spine as shown by three-dimensional load-displacement curves. J Bone Joint Surg Am 76: 413-424, 1994.

31. Wilke HJ, Neef P, Caimi M, Hoogland T and Claes LE: New in vivo measurements of pressures in the intervertebral disc in daily life. Spine (Phila Pa 1976) 24: 755-762, 1999.

32. Rohlmann A, Zander T, Schmidt H, Wilke HJ and Bergmann G: Analysis of the influence of disc degeneration on the mechanical behaviour of a lumbar motion segment using the finite element method. J Biomech 39: 2484-2490, 2006.

33. Ng HW and Teo EC: Nonlinear finite-element analysis of the lower cervical spine (C4-C6) under axial loading. J Spinal Disord 14: 201-210, 2001.

34. Smit TH, Odgaard A and Schneider E: Structure and function of vertebral trabecular bone. Spine (Phila Pa 1976) 22: 2823-2833, 1997.

35. Pitzen T, Geisler F, Matthis D, Müller-Storz H, Barbier D, Steudel WI and Feldges A: A finite element model for predicting the biomechanical behaviour of the human lumbar spine. Control Engineering Practice 10: 83-90, 2002.

36. Chen CS, Cheng CK, Liu CL and Lo WH: Stress analysis of the disc adjacent to interbody fusion in lumbar spine. Med Eng Phys 23: 483-491, 2001. 